\title{
UNIFORMITY AND UNIFORMLY CONTINUOUS FUNCTIONS FOR LOCALLY COMPACT GROUPS
}

\author{
PAUL MILNES
}

(Communicated by Jonathan M. Rosenberg)

\begin{abstract}
We show that a locally compact group $G$ has equivalent right and left uniform structures if (and only if) the sets of bounded, complex-valued, right and left uniformly continuous functions on $G$ coincide. Along the way it is seen that $G$ has equivalent right and left uniform structures if (and only if) each $\sigma$-compact subgroup of $G$ has equivalent right and left uniform structures. We also note that a bounded function $f: G \rightarrow \mathbb{C}$ is right uniformly continuous if (and only if) $\left.f\right|_{H}$ is right uniformly continuous for each $\sigma$ compact subgroup $H$ of $G$. $\sigma$-compactness cannot be weakened to compact generation for these last results; a $\sigma$-compact group is exhibited which has inequivalent right and left uniform structures, and for which each compactly generated subgroup has equivalent right and left uniform structures.
\end{abstract}

For the precise definition of the right and left uniform structures on a locally compact group $G$ we refer to [2, p. 21], noting here only that $G$ has equivalent right and left uniform structures (i.e., $G$ is equistructural ) if and only if, whenever $\left\{s_{\alpha}\right\}$ and $\left\{t_{\alpha}\right\}$ are nets in $G$ with $s_{\alpha}^{-1} t_{\alpha} \rightarrow e$, the identity of $G$, it follows necessarily that $s_{\alpha} t_{\alpha}^{-1} \rightarrow e$. A function $f: G \rightarrow \mathbb{C}$ is called right (left) uniformly continuous if, for every $\epsilon>0$, there is a neighbourhood $V$ of $e$ such that $|f(s)-f(t)|<\epsilon$ if $s t^{-1} \in V\left(s^{-1} t \in V\right)$. We denote the set of bounded right (left) uniformly continuous functions on $G$ by $\mathscr{U}_{R}(G)\left(\mathscr{U}_{L}(G)\right)$. Of course, $\mathscr{U}_{R}(G)=\mathscr{U}_{L}(G)$ if $G$ is equistructural.

We prove our main result first in the metric setting.

Proposition 1. For metric locally compact $G, G$ is equistructural if (and only if)

$$
\mathscr{U}_{R}(G)=\mathscr{U}_{L}(G) .
$$

Proof. Suppose $G$ is not equistructural. Then there exist sequences $\left\{s_{n}\right\}$ and $\left\{t_{n}\right\}$ with $s_{n}^{-1} t_{n} \rightarrow e$ and, without loss of generality, $\left\{s_{n} t_{n}^{-1}\right\} \cap V=\phi$ for some compact symmetric neighbourhood $V$ of $e$. Clearly $s_{n} \rightarrow \infty$ and $t_{n} \rightarrow \infty$, so

Received by the editors December 12, 1988 and, in revised form, November 22, 1989.

1980 Mathematics Subject Classification (1985 Revision). Primary 22D05.

Key words and phrases. Locally compact group, uniform structure, uniformly continuous function.

This research was supported in part by NSERC grant A7857. 
we may assume that $V t_{n} \cap V t_{m}=\phi$ if $n \neq m$. We construct a subsequence of $\left\{\left(s_{n}, t_{n}\right)\right\}$. Let $\left(s_{n_{1}}, t_{n_{1}}\right)=\left(s_{1}, t_{1}\right)$. Let $\left(s_{n_{2}}, t_{n_{2}}\right)$ be such that

$$
s_{n_{2}} t_{n_{1}}^{-1} \notin V, \quad s_{n_{1}} t_{n_{2}}^{-1} \notin V
$$

(which is possible, since $s_{n} \rightarrow \infty, t_{n} \rightarrow \infty$ ). Hence, by induction we can choose $\left(s_{n_{j}}, t_{n_{j}}\right)$ such that

$$
s_{n_{j}} t_{n_{k}}^{-1}, s_{n_{k}} t_{n_{j}}^{-1} \notin V, \quad 1 \leqslant k<j .
$$

Put $\left(u_{j}, v_{j}\right)=\left(s_{n_{j}}, t_{n_{j}}\right)$. Let $h \in \mathscr{U}_{R}(G)$ satisfy $0 \leqslant h \leqslant 1, h(e)=1, h=0$ off $V$. Then

$$
0 \leqslant f:=\sum_{j=1}^{\infty} R_{v_{j}^{-1}} h \leqslant 1
$$

( $R_{v} h$ being defined by $R_{v} h(s):=h(s v)$ ), since $V t_{n} \cap V t_{m}=\phi$ if $n \neq m$, and $f \in \mathscr{U}_{R}(G)$ (see [1, 4.4.16], for example). But $f\left(u_{j}\right)=0$ and $f\left(v_{j}\right)=1$ for all $i$, while $u_{j}^{-1} v_{j} \rightarrow e$, i.e., $f \notin \mathscr{U}_{L}(G)$.

Theorem 2. For locally compact $G, G$ is equistructural if (and only if) $\mathscr{U}_{R}(G)=$ $\mathscr{U}_{L}(G)$.

Proof. As in Proposition 1, suppose $G$ is not equistructural. Then there exist nets $\left\{s_{\alpha}\right\}$ and $\left\{t_{\alpha}\right\}$ in $G$ such that $s_{\alpha}^{-1} t_{\alpha} \rightarrow e$ and $\left\{s_{\alpha} t_{\alpha}^{-1}\right\} \cap V=\phi$ for some compact symmetric neighbourhood $V$ of $e$. Set $K_{n}=\bigcup_{j=1}^{n} V^{j}$ for all $n \in \mathbb{N}$. We extract a sequence from $\left\{s_{\alpha}^{-1} t_{\alpha}\right\}$.

Pick $\alpha_{1}$ so that $s_{\alpha_{1}}^{-1} t_{\alpha_{1}} \in V_{1}:=V$, and put $s_{1}=s_{\alpha_{1}}$ and $t_{1}=t_{\alpha_{1}}$. Set $A_{1}:=\left\{s_{1}, t_{1}, e, s_{1}^{-1}, t_{1}^{-1}\right\}$. The set $B_{1}:=A_{1} K_{1}$ is compact. Because of the continuity of multiplication in $G$, we can pick a compact symmetric neighbourhood $V_{2}$ of $e$ such that $V_{2}^{2} \subset V_{1}$ and $s V_{2} s^{-1} \subset V_{1}$ for all $s \in B_{1}$. Then pick $s_{2}^{-1} t_{2}:=s_{\alpha_{2}}^{-1} t_{\alpha_{2}} \in V_{2}$. Thus we get by induction $s_{n}^{-1} t_{n}:=s_{\alpha_{n}}^{-1} t_{\alpha_{n}} \in V_{n}$ and compact symmetric neighbourhood $V_{n+1}$ of $e$ such that $V_{n+1}^{2} \subset V_{n}$ and $s V_{n+1} s^{-1} \subset V_{n}$ for all $s$ in the compact set $B_{n}:=\left(A_{n} K_{n}\right)^{n}$, where $A_{n}$ is the set of all products of $n$ or fewer members of $\left\{s_{j}, t_{j}, s_{j}^{-1}, t_{j}^{-1} \mid 1 \leqslant j \leqslant n\right\}$. Then $G_{1}:=\bigcup_{n=1}^{\infty}\left(A_{n} K_{n}\right)^{n}$ is an open $\sigma$-compact subgroup of $G, K=\bigcap_{n=1}^{\infty} V_{n}$ is a compact normal subgroup of $G_{1}$ and $G_{1} / K$ is metrizable. (See [4, p. 58] for more on these ideas.) Also, if $\pi: G_{1} \rightarrow G_{1} / K$ is the quotient map, we have

$$
\pi\left(s_{n}^{-1} t_{n}\right)=\pi\left(s_{n}\right)^{-1} \pi\left(t_{n}\right) \rightarrow e \in G_{1} / K
$$

and further, $s_{n} t_{n}^{-1} K \cap V_{2} K \subset s_{n} t_{n}^{-1} V_{3} \cap V_{2} V_{3}=\phi$, so $\left\{\pi\left(s_{n}\right) \pi\left(t_{n}\right)^{-1}\right\} \cap \pi\left(V_{2}\right)=\phi$, $\pi\left(V_{2}\right)$ being a compact neighbourhood of $e \in G_{1} / K$.

Now, as in Proposition 1 , let $\left\{\left(u_{j}, v_{j}\right)\right\}$ be a subsequence of $\left\{\left(\pi\left(s_{n}\right), \pi\left(t_{n}\right)\right)\right\}$ and let

$h \in \mathscr{U}_{R}\left(G_{1} / K\right) . \quad 0 \leqslant h \leqslant 1, \quad h(e)=1, \quad h=0 \quad$ off $\pi\left(V_{2}\right)$, 
so that

$$
f_{1}:=\sum_{j=1}^{\infty} R_{v_{j}^{-1}} h \in \mathscr{U}_{R}\left(G_{1} / K\right) \backslash \mathscr{U}_{L}\left(G_{1} / K\right) .
$$

Set $f:=f_{1} \circ \pi \in \mathscr{U}_{R}\left(G_{1}\right)$. We consider $f$ to be in $\mathscr{U}_{R}(G)$ by defining it to be 0 off $G_{1}$. We must show $f \notin \mathscr{U}_{L}(G)$. For each $j$, let $x_{j}$ and $y_{j}^{\prime}$ be members of the original nets $\left\{s_{\alpha}\right\}$ and $\left\{t_{\alpha}\right\}$, respectively, such that $\pi\left(x_{j}\right)=u_{j}$ and $\pi\left(y_{j}^{\prime}\right)=v_{j}$. Each $x_{j}^{-1} y_{j}^{\prime} \in V_{j}$; let $\left\{x_{j_{\delta}}^{-1} y_{j_{\delta}}^{\prime}\right\}$ be a convergent subnet of $\left\{x_{j}^{-1} y_{j}^{\prime}\right\}$ with limit $z$, say, which must be in $K$. Put $y_{j}=y_{j}^{\prime} z^{-1}$ for all $j$. Then $x_{j_{\delta}}^{-1} y_{j_{\delta}} \rightarrow e$, but

$$
\left|f\left(x_{j_{\delta}}\right)-f\left(y_{j_{\delta}}\right)\right|=\left|f_{1}\left(u_{j_{\delta}}\right)-f_{1}\left(v_{j_{\delta}}\right)\right|=1
$$

for all $\delta$ (since $\left.\pi\left(y_{j}\right)=\pi\left(y_{j}^{\prime}\right)\right)$, i.e., $f \notin \mathscr{U}_{L}(G)$.

Remarks and examples. 1. The proof of Theorem 2 shows that $G$ is equistructural if each of its open $\sigma$-compact subgroups is equistructural. Using the same general ideas, one can show that a function $f: G \rightarrow \mathbb{C}$ is in $\mathscr{U}_{L}(G)$ if $\left.f\right|_{H} \in \mathscr{U}_{L}(H)$ for each open $\sigma$-compact subgroup $H$ of $G$.

2. Let the symmetric group $S_{\infty}$ act in the obvious way on $\mathbb{T}^{\mathbb{N}}$ : for each $s \in$ $s_{\infty}$ and $h=\left(h_{n}\right) \in \mathbb{T}^{\mathbb{N}}, s(h)=\left(h_{s(n)}\right)$, where $s: \mathbb{N} \rightarrow \mathbb{N}$ is $1-1$ with $s(n)=n$ for all but finitely many $n$. Then, for the semidirect product $G=\mathbb{T}^{\mathbb{N}} \times S_{\infty}$ with product

$$
\left(h^{\prime}, s^{\prime}\right)(h, s)=\left(h^{\prime} s^{\prime}(h), s^{\prime} s\right),
$$

every compactly generated subgroup $H$ is compact, hence all uniformities generating the topology of $H$ are equivalent. However, the function $f:(h, s) \rightarrow$ $h_{1}$ is in $\mathscr{U}_{R}(G) \backslash \mathscr{U}_{L}(G)$.

3. A space with the same set of bounded uniformly continuous functions for each of two different uniformities is easy enough to come by. On $\mathbb{R}$ consider Unif $f_{1}$ generated by the usual metric and $U n i f_{2}$ generated by the pseudometrics $d_{f}(s, t)=|f(s)-f(t)|$, where $f \in \mathscr{U}_{R}(G)$ (i.e., $f$ is uniformly continuous with respect to $\left.U n i f_{1}\right)$. Then the bounded functions on $\mathbb{R}$ uniformly continuous with respect to $U n i f_{1}$ coincide with those uniformly continuous with respect to $U n i f_{2}$. But these uniformities are quite different: with $U n i f_{1} \mathbb{R}$ is complete but not totally bounded, while with $U n i f_{2} \mathbb{R}$ is totally bounded but not complete. Also $U n i f_{1}>U n i f_{2}$, i.e., Unif $f_{1}$ is stronger than $U n i f_{2}$. It is now easy to see how to get noncomparable uniformities with the same bounded uniformly continuous functions. Let $X=\mathbb{R}_{1} \cup \mathbb{R}_{2}$ be a disjoint union of two copies of $\mathbb{R}$. Here are noncomparable uniformities on $X$ :

(1) Unif on $\mathbb{R}_{1}$ and Unif on $\mathbb{R}_{2}$.

(2) Unif $f_{2}$ on $\mathbb{R}_{1}$ and Unif on $\mathbb{R}_{2}$.

But $X$ has the same bounded uniformly continuous functions with (1) or (2). (We point out that $\mathbb{R}$ with $U n i f_{2}$ is just the precompact reflection [3, p. 23] of $\mathbb{R}$ with Unif $_{1}$; thus the argument given here applies in a quite general setting.) 
Note of the referee. A pseudometric is left uniformly continuous if and only if its restriction to each $\sigma$-compact subgroup is left uniformly continuous.

\section{BIBLIOGRAPHY}

1. J. F. Berglund, H. D. Junghenn, and P. Milnes, Analysis on semigroups: function spaces, compactifactions, representations, Wiley, New York, 1989.

2. E. Hewitt and K. A. Ross, Abstract harmonic analysis I, Springer-Verlag, New York, 1963.

3. J. R. Isbell, Uniform spaces, Amer. Math. Soc., Providence, RI, 1964.

4. D. Montgomery and L. Zippin, Topological transformation groups, Interscience, New York, 1955.

Department of Mathematics, University of Western Ontario, London, Ontario N6a 5B7 CANADA 